\title{
Reconfigurable Intelligent Surface for Interference Alignment in MIMO Device-to-Device Networks
}

\author{
Min $\mathrm{Fu}^{* \dagger \S}$, Yong Zhou*, and Yuanming Shi* \\ * School of Information Science and Technology, ShanghaiTech University, Shanghai 201210, China \\ †Shanghai Institute of Microsystem and Information Technology, Chinese Academy of Sciences, China \\ $\S$ University of Chinese Academy of Sciences, Beijing 100049, China \\ Email: \{fumin, zhouyong, shiym\}@ shanghaitech.edu.cn
}

\begin{abstract}
In multiple-input multiple-output (MIMO) deviceto-device (D2D) networks, interference and rank-deficient channels are the critical bottlenecks for achieving high degrees of freedom (DoFs). In this paper, we propose a reconfigurable intelligent surface (RIS) assisted interference alignment strategy to simultaneous mitigate the co-channel interference and cope with rank-deficient channels, thereby improving the feasibility of interference alignment conditions and in turn increasing the achievable DoFs. The key enabler is a general low-rank optimization approach that maximizes the achievable DoFs by jointly designing the phase-shift and transceiver matrices. To address the unique challenges of the coupled optimization variables, we develop a block-structured Riemannian pursuit method by solving fixed-rank and unit modulus constrained least square subproblems along with rank increase. Finally, to reduce the computational complexity and achieve good DoF performance, we develop unified Riemannian conjugate gradient algorithms to alternately optimize the fixed-rank transceiver matrix and the unit modulus constrained phase shifter by exploiting the non-compact Stiefel manifold and the complex circle manifold, respectively. Numerical results demonstrate the effectiveness of deploying an RIS and the superiority of the proposed block-structured Riemannian pursuit method in terms of the achievable DoFs and the achievable sum rate.
\end{abstract}

\section{INTRODUCTION}

Device-to-device (D2D) communication has been recognized as a promising technology that can enhance the efficiency of wireless networks by allowing direct communication between devices in proximity without going through base stations [1]. However, interference is one of the critical issues in emerging D2D networks, where the co-located transceiver pairs may severely interfere with each other [2]. Fortunately, interference alignment [3] as a powerful technology has been well studied to manage interference in various interference-limited scenarios (e.g., multiple-input multiple-output (MIMO) beamforming [3]). The main idea is to restrict the desired signal space being a complement to the interference space. Such a linear interference management strategy achieves the optimal degrees of freedom (DoFs) of generic channels in high signal-to-noise ratio (SNR) [3]. However, due to poor scattering, insufficient antenna-spacing, and keyhole effects, rank-deficient channels lead to significant performance degradation in terms of achievable DoFs [4].

Reconfigurable intelligent surfaces (RISs) as a costeffective technology have recently been proposed to enhance the network performance [5]-[7]. Specifically, an RIS consists of a number of passive elements, each of which is able to dynamically re-scatter the incident signals with the desired phase shift via a smart controller, thereby combating the unfavorable propagation conditions. There is a growing body of recent works to study the performance of various RISassisted wireless networks. The advantages of RIS were leveraged to reduce the energy consumption [8], [9] and increase the achievable data rate [10]-[12]. In addition, various channel estimation methods were proposed to estimate channel coefficients of RIS-related links (e.g., compressivesensing based methods [13]). However, it is still not clear whether the feasibility of interference alignment conditions can be improved by RIS. This motivates us to explore the benefits of RIS to improve the feasibility of interference alignment conditions in D2D networks and in turn increase the DoFs without the assumption of independent channels.

In this paper, we consider an RIS-assisted MIMO D2D network, where the RIS is proposed to assist interference alignment. Specifically, we jointly design the phase-shift matrix at RIS and the transceiver matrix to maximize the achievable DoFs. By establishing interference alignment conditions, we develop a rank minimization problem to maximize DoFs. However, the formulated problem is highly intractable due to the non-convexity of the bilinear function, rank function, and the unit modulus constraints. To address this unique challenges, we propose a block-structured Riemannian pursuit method by solving a sequence of non-convex least square subproblems along with rank increase. To deal with the coupled fixed-rank transceiver matrix and unit modulus constrained phase shifters, we further develop unified low-complexity Riemannian conjugate gradient (RCG) methods with good performance [14], [15] to alternately optimize transceiver matrix and phase shifters by exploiting the non-compact Stiefel manifold and complex circle manifold, respectively. Simulation results validate the effectiveness of deploying an RIS and the performance gains of the proposed method in terms of the achievable DoFs and the achievable sum rate.

Organization: The remainder of this paper is organized as follows. Section II describes the system model and problem formulation. We present a block-structured Riemannian pursuit method to solve the formulated problem in Section III. Section IV presents the numerical results and Section V concludes this paper. 


\section{System Model ANd Problem Formulation}

\section{A. System Model}

We consider an MIMO D2D network consisting of $K$ transceiver pairs with the assist of an RIS. We denote $\mathcal{K}=$ $\{1, \ldots, K\}$ as the index set of D2D pairs. The $k$-th transmitter and receiver are equipped with $N_{k}$ and $M_{k}$ antennas, respectively. With full frequency reuse, the D2D pairs may severely interfere with each other [1], [2]. Hence, we propose to deploy an RIS equipped with $L$ passive reflecting elements, aiming to alleviate the severe co-channel interference among $K$ pairs. We denote $\boldsymbol{\Theta}=\operatorname{diag}\left\{v_{1}, \ldots, v_{L}\right\} \in \mathbb{C}^{L \times L}$ as the diagonal reflection matrix of the RIS. Specifically, let $v_{l} \in \mathbb{C}$ denote the reflection coefficient of the $l$-th RIS element, which is assumed to satisfy $\left|v_{l}\right|=1, \forall l$ [8]-[12].

We denote $\boldsymbol{H}_{i j} \in \mathbb{C}^{M_{i} \times N_{j}}$ as the channel of direct link between the $i$-th receiver and the $j$-th transmitter, $\boldsymbol{R}_{i} \in$ $\mathbb{C}^{M_{i} \times L}$ as that between the RIS and the $i$-th receiver, and $\boldsymbol{T}_{j} \in \mathbb{C}^{L \times N_{j}}$ as that between the $j$-th transmitter and the RIS. Thus, the composite channel matrix between the $i$-th receiver and the $j$-th transmitter, consisting of both the direct and reflect links, is given by $\tilde{\boldsymbol{H}}_{i j}=\boldsymbol{H}_{i j}+\boldsymbol{R}_{i} \boldsymbol{\Theta} \boldsymbol{T}_{j} \in \mathbb{C}^{M_{i} \times N_{j}}$.

Each transmitter $i$ has a message $W_{i}$ intended for receiver $i$. Message $W_{i}$ is encoded into a vector $\boldsymbol{x}_{i} \in \mathbb{C}^{r N_{i}}$ of length $r N_{i}$ and transmitted by $N_{i}$ antennas over $r$ channel uses, where $\mathbb{E}\left[\boldsymbol{x}_{i}^{\mathrm{H}} \boldsymbol{x}_{i}\right] \leq P$. We consider the quasi-static fading channels, i.e., $\boldsymbol{H}_{i j}, \boldsymbol{R}_{i}$, and $\boldsymbol{T}_{j}$ remain invariant over $r$ channel uses during the data transmission. Hence, the phaseshift matrix at the RIS is designed to be invariant during the data transmission. Hence, the signal received by the $m$-th antenna of receiver $i$ over $r$ channel uses is given by

$$
\begin{aligned}
\boldsymbol{y}_{i}[m] & =\sum_{j=1}^{K} \sum_{n=1}^{N_{j}}\left(H_{i j}[m, n]+\boldsymbol{R}_{i}[m] \boldsymbol{\Theta} \boldsymbol{T}_{j}[n]\right) \boldsymbol{x}_{j}[n]+\boldsymbol{z}_{i}[m] \\
& =\sum_{j=1}^{K} \sum_{n=1}^{N_{j}} \tilde{H}_{i j}[m, n] \boldsymbol{x}_{j}[n]+\boldsymbol{z}_{i}[m],
\end{aligned}
$$

where $\boldsymbol{z}_{i}[m] \in \mathbb{C}^{r} \sim \mathcal{C N}\left(\mathbf{0}, \sigma \boldsymbol{I}_{r}\right)$ is the additive white Gaussian noise (AWGN), $\boldsymbol{x}_{j}[n] \in \mathbb{C}^{r}$ corresponds to the signal transmitted by the $n$-th antenna at transmitter $j$ over $r$ channel uses, $\boldsymbol{y}_{i}[m] \in \mathbb{C}^{r}$ is the signal received by the $m$-th antenna at receiver $i$ over $r$ channel uses, $\tilde{H}_{i j}[m, n]$ is the $(m, n)$-th entry of matrix $\tilde{\boldsymbol{H}}_{i j}, H_{i j}[m, n]$ is the $(m, n)$ th entry of matrix $\boldsymbol{H}_{i j}, \boldsymbol{R}_{i}[m] \in \mathbb{C}^{1 \times L}$ is the $m$-th row of matrix $\boldsymbol{R}_{i}$, and $\boldsymbol{T}_{j}[n] \in \mathbb{C}^{L}$ is the $n$-th column of matrix $\boldsymbol{T}_{j}$.

The achieve rate $R\left(W_{i}\right)$ is achievable of message $W_{i}$ if there exists an encoding scheme such the message $W_{i}$ is $R\left(W_{i}\right)$ while the error probability of decoding $W_{i}$ for user $i$ can be made arbitrarily small as the number of channel uses $r$ is big enough. For each message delivery, the DoF is characterized by the first order characterization of channel capacity and is defined by [3], [16]

$$
\operatorname{DoF}\left(W_{i}\right)=\lim _{\mathrm{SNR}_{i} \rightarrow \infty} \frac{R\left(W_{i}\right)}{\log \left(\mathrm{SNR}_{i}\right)}, \forall i \in \mathcal{K},
$$

where $\mathrm{SNR}_{i}$ denotes the $i$-th transmitter's SNR. We aim to design an effective linear interference alignment strategy to maximize the achievable DoFs in the sequel.

\section{B. Interference Alignment}

Linear interference alignment have attracted considerable attention to manage the co-channel interference due to their low-complexity and the DoF optimality in high SNR scenarios [3]. Specifically, let the linear precoding matrix at transmitter $i$ and decoding matrix at receiver $i$ over $r$ channel uses be $\boldsymbol{V}_{i} \in \mathbb{C}^{N_{i} r \times d_{i}}$ and $\boldsymbol{U}_{i} \in \mathbb{C}^{M_{i} r \times d_{i}}$, respectively. Message $W_{i}$ is split into $d_{i}$ independent scalar data streams, denoted as $s_{i} \in \mathbb{C}^{d_{i}}$, and is transmitted along with the precoding matrix $\boldsymbol{V}_{i}$, i.e., $\boldsymbol{x}_{i}=\boldsymbol{V}_{i} \boldsymbol{s}_{i}$. We follow [2], and assume that the full CSI is known and a centralized node is responsible for the network optimization and then the feedback of the optimization parameters to the $\mathrm{D} 2 \mathrm{D}$ pairs. Note that even with this ideal assumption, this kind of optimization problems is still NP-hard. Based on the aforementioned definitions, the received signal $\boldsymbol{y}_{i} \in \mathbb{C}^{r M_{i}}$ at receiver $i$ is given by

$$
\boldsymbol{y}_{i}=\left(\tilde{\boldsymbol{H}}_{i i} \otimes \boldsymbol{I}_{r}\right) \boldsymbol{V}_{i} \boldsymbol{s}_{i}+\sum_{i \neq j}\left(\tilde{\boldsymbol{H}}_{i j} \otimes \boldsymbol{I}_{r}\right) \boldsymbol{V}_{j} \boldsymbol{s}_{j}+\boldsymbol{z}_{i},
$$

where $\otimes$ is the Kronecker product operator and $\boldsymbol{z}_{i} \sim$ $\mathcal{C N}\left(\mathbf{0}, \sigma \boldsymbol{I}_{r M_{i}}\right)$. With decoding matrix $\boldsymbol{U}_{i}$, we have

$$
\tilde{\boldsymbol{y}}_{i}=\boldsymbol{U}_{i}^{\mathrm{H}}\left(\tilde{\boldsymbol{H}}_{i i} \otimes \boldsymbol{I}_{r}\right) \boldsymbol{V}_{i} \boldsymbol{s}_{i}+\boldsymbol{U}_{i}^{\mathrm{H}} \sum_{i \neq j}\left(\tilde{\boldsymbol{H}}_{i j} \otimes \boldsymbol{I}_{r}\right) \boldsymbol{V}_{j} \boldsymbol{s}_{j}+\tilde{\boldsymbol{z}}_{i}(4)
$$

where $\tilde{\boldsymbol{y}}_{i} \in \mathbb{C}^{d_{i}}, \tilde{\boldsymbol{z}}_{i}=\boldsymbol{U}_{i}^{\mathrm{H}} \boldsymbol{z}_{i} \in \mathbb{C}^{d_{i}}$. We observe that $\tilde{\boldsymbol{y}}_{i}$ can be decomposed into desired signal, interference, and noise. In the high SNR regime, we exploit the interference alignment to design the precoding, decoding, and phase-shifter matrices to cancel interference while preserving the desired signals, which imposes the following conditions [3]

$$
\begin{aligned}
\boldsymbol{U}_{i}^{\mathrm{H}}\left(\tilde{\boldsymbol{H}}_{i j} \otimes \boldsymbol{I}_{r}\right) \boldsymbol{V}_{j} & =\mathbf{0}, \forall i \neq j, \\
\operatorname{rank}\left(\boldsymbol{U}_{i}^{\mathrm{H}}\left(\tilde{\boldsymbol{H}}_{i i} \otimes \boldsymbol{I}_{r}\right) \boldsymbol{V}_{i}\right) & =d_{i}, \forall i=1, \ldots, K .
\end{aligned}
$$

Equation (5) guarantees all the interfering signals at receiver $i$ lie in the subspace orthogonal to $\boldsymbol{U}_{i}$, while Equation (6) assures that the signal subspace $\left(\tilde{\boldsymbol{H}}_{i i} \otimes \boldsymbol{I}_{r}\right) \boldsymbol{V}_{i}$ has dimension $d_{i}$ and is linearly independent of the interference subspace. If conditions (5) and (6) are satisfied, the parallel interferencefree channels can be obtained over $r$ channel uses. Therefore, the DoF tuple $\left(d_{1} / r, \ldots, d_{K} / r\right)$ is achievable for messages $\left\{W_{1}, \ldots, W_{K}\right\}$.

\section{Problem Formulation}

For fixed $\left\{d_{1}, \ldots, d_{K}\right\}$, the smaller the value of $r$, the larger the achievable DoFs. As a result, we formulate a rank minimization problem to maximize the achievable DoFs in this subsection. We first develop a low-rank model to find the minimal channel uses $r$ such that the interference alignment conditions (5) and (6) are feasible. We note that

$$
\boldsymbol{U}_{i}^{\mathrm{H}}\left(\tilde{\boldsymbol{H}}_{i j} \otimes \boldsymbol{I}_{r}\right) \boldsymbol{V}_{j}=\sum_{m=1}^{M_{i}} \sum_{n=1}^{N_{j}} \tilde{H}_{i j}[m, n] \boldsymbol{U}_{i}^{\mathrm{H}}[m] \boldsymbol{V}_{j}[n],
$$


where $\boldsymbol{V}_{j}[n] \in \mathbb{C}^{r \times d_{j}}$ and $\boldsymbol{U}_{i}[m] \in \mathbb{C}^{r \times d_{i}}$ correspond to the $n$-th antenna of transmitter $j$ and the $m$-th antenna of receiver $i$ over $r$ channel uses, respectively. We define $\boldsymbol{X}_{i, j}[m, n]=\boldsymbol{U}_{i}^{\mathrm{H}}[m] \boldsymbol{V}_{j}[n] \in \mathbb{C}^{d_{i} \times d_{j}}$. The rank constraints in (6) are represented by its column-reduced echelon form, i.e., $\boldsymbol{U}_{i}^{\mathrm{H}}\left(\tilde{\boldsymbol{H}}_{i i} \otimes \boldsymbol{I}_{r}\right) \boldsymbol{V}_{i}=\boldsymbol{I}$ [3], [16] to support efficient algorithm design. Thus, conditions (5) and (6) can be rewritten as

$$
\begin{aligned}
& \sum_{m=1}^{M_{i}} \sum_{n=1}^{N_{j}} \tilde{H}_{i j}[m, n] \boldsymbol{X}_{i, j}[m, n]=\mathbf{0}, \forall i \in \mathcal{K}, i \neq j,(8) \\
& \sum_{m=1}^{M_{i}} \sum_{n=1}^{N_{i}} \tilde{H}_{i i}[m, n] \boldsymbol{X}_{i, i}[m, n]=\boldsymbol{I}, \forall i \in \mathcal{K}
\end{aligned}
$$

Let $M=\sum_{i=1}^{K} M_{i} d_{i}, N=\sum_{j=1}^{K} N_{j} d_{j}$, and $S=$ $\sum_{i=1}^{K} \sum_{j=1}^{K} d_{i} d_{j}$. By defining a set of matrices

$$
\begin{aligned}
\tilde{\boldsymbol{V}}_{j} & =\left[\boldsymbol{V}_{j}[1], \ldots, \boldsymbol{V}_{j}\left[N_{j}\right]\right], \tilde{\boldsymbol{U}}_{i}=\left[\boldsymbol{U}_{i}[1], \ldots, \boldsymbol{U}_{i}\left[M_{i}\right]\right], \\
\tilde{\boldsymbol{V}} & =\left[\tilde{\boldsymbol{V}}_{1}, \ldots, \tilde{\boldsymbol{V}}_{K}\right], \tilde{\boldsymbol{U}}=\left[\tilde{\boldsymbol{U}}_{1}, \ldots, \tilde{\boldsymbol{U}}_{K}\right],
\end{aligned}
$$

where $\tilde{\boldsymbol{V}}_{j} \in \mathbb{C}^{r \times N_{j} d_{j}}, \tilde{\boldsymbol{U}}_{i} \in \mathbb{C}^{r \times M_{i} d_{i}}, \tilde{\boldsymbol{V}}_{1} \in \mathbb{C}^{r \times N}$, and $\tilde{\boldsymbol{U}} \in \mathbb{C}^{r \times M}$, we further have

$$
\begin{aligned}
\boldsymbol{X}_{i, j} & =\left[\boldsymbol{X}_{i, j}[m, n]\right]=\tilde{\boldsymbol{U}}_{i}^{\mathrm{H}} \tilde{\boldsymbol{V}}_{j} \in \mathbb{C}^{M_{i} d_{i} \times N_{j} d_{j}}, \\
\boldsymbol{X} & =\left[\boldsymbol{X}_{i, j}\right]=\tilde{\boldsymbol{U}}^{\mathrm{H}} \tilde{\boldsymbol{V}} \in \mathbb{C}^{M \times N} .
\end{aligned}
$$

Note that the rank of matrix $\boldsymbol{X}$ is equal to the number of channel uses $r$ since $\boldsymbol{X}=\tilde{\boldsymbol{U}}^{\mathrm{H}} \tilde{\boldsymbol{V}}$, i.e., $\operatorname{rank}(\boldsymbol{X})=r$. We vectorize both sides of (8) and (9), followed by characterizing both equations as $\mathcal{A}(\boldsymbol{X}, \boldsymbol{\Theta})=\boldsymbol{b}$ with the bilinear operator $\mathcal{A}: \mathbb{C}^{M \times N} \mapsto \mathbb{C}^{S}$ as a function of $\left\{\boldsymbol{H}_{i j}, \boldsymbol{R}_{i}, \boldsymbol{T}_{j}\right\}$. We hence propose the following generalized low-rank optimization problem to maximize the achievable DoFs

$$
\begin{aligned}
\mathscr{P}: \underset{\boldsymbol{X}, \boldsymbol{\Theta}}{\operatorname{minimize}} & \operatorname{rank}(\boldsymbol{X}) \\
\text { subject to } & \mathcal{A}(\boldsymbol{X}, \boldsymbol{\Theta})=\boldsymbol{b}, \\
& \left|v_{l}\right|=1, \forall l=1, \ldots, L .
\end{aligned}
$$

However, Problem $\mathscr{P}$ is computationally difficult due to the non-convexity of the rank function, the bilinear equation constraint, and the unit modulus constraints. General convex relaxations (e.g., nuclear norm for rank) for Problem $\mathscr{P}$ are inapplicable due to the bilinear constraint and the unit modulus constraints. In the following section, we propose a block-structured Riemannian pursuit method to solve $\mathscr{P}$ in the manifold space to achieve the algorithmic advantages and admirable performance.

\section{Block-STRUCtURed Riemannian PURsuit}

In this section, we develop a block-structured Riemannian pursuit for the rank minimization problem in the RIS-assisted MIMO D2D networks to reduce the computational complexity and achieve good performance.

\section{A. Rank Pursuit}

In this subsection, we present an efficient rank pursuit strategy to solve Problem $\mathscr{P}$ by alternately solving the fixedrank optimization and rank increase, thereby detecting the minimum rank of matrix $\boldsymbol{X}$. Specifically, fixing the rank of

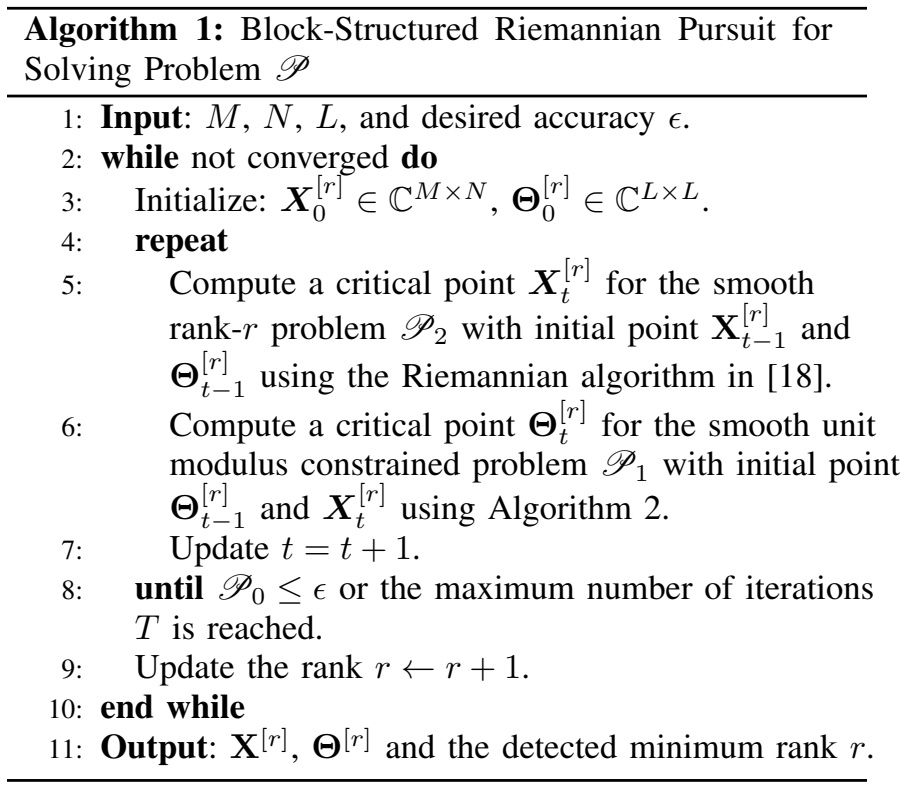

matrix $\boldsymbol{X}$ as $r$, we need to solve the following non-convex optimization problem

$$
\begin{array}{cl}
\underset{\boldsymbol{X}, \boldsymbol{\Theta}}{\operatorname{minimize}} & f_{0}(\boldsymbol{X}, \boldsymbol{\Theta})=\frac{1}{2}\|\mathcal{A}(\boldsymbol{X}, \boldsymbol{\Theta})-\boldsymbol{b}\|_{2}^{2} \\
\text { subject to } & \operatorname{rank}(\boldsymbol{X})=r, \\
& \left|v_{l}\right|=1, \forall l=1, \ldots, L .
\end{array}
$$

Although the problem (13) is still non-convex, we observe that the objective function $f_{0}$ is smooth about each block variable which stays different manifold spaces, i.e., fixedrank complex matrix in non-compact Stiefel manifold and unit modulus constrained phase shifters in the complex circle manifold. The low computational complexity and good performance of Riemannian optimization [14], [15] have been validated in various wireless networks e.g., topological interference management [16]-[18], and blind demixing [19]. Hence, we decouple the variables into two blocks, following by adopting unified RCG algorithms to design the transceiver and phase-shift matrices on the manifold space alternately. The proposed algorithm is summarized in Algorithm 1.

\section{B. Optimizing Block $\Theta$}

We define $\boldsymbol{v}=\left[v_{1}^{\mathrm{H}}, \ldots, v_{L}^{\mathrm{H}}\right]^{\mathrm{H}} \in \mathbb{C}^{L}$. By denoting $\boldsymbol{B}_{i j}[m, n]=H_{i j}[m, n] \boldsymbol{x}_{i j}[m, n] \in \mathbb{C}^{d_{i} \times d_{j}}$ and $\boldsymbol{a}_{i j}[m, n]=$ $\boldsymbol{R}_{i}[m] \operatorname{diag}\left(\boldsymbol{T}_{j}[n]\right) \in \mathbb{C}^{1 \times L}$, given fixed block $\boldsymbol{X}$, we have

$$
\begin{gathered}
\left(H_{i j}[m, n]+\boldsymbol{R}_{i}[m] \boldsymbol{\Theta} \boldsymbol{T}_{j}[n]\right) \boldsymbol{X}_{i j}[m, n] \\
\quad=\boldsymbol{B}_{i j}[m, n]+\boldsymbol{a}_{i j}[m, n] \boldsymbol{v} \boldsymbol{X}_{i j}[m, n] .
\end{gathered}
$$

The conditions (8) and (9) can be rewritten as

$$
\begin{aligned}
\sum_{m=1}^{M_{i}} \sum_{n=1}^{N_{j}} \boldsymbol{B}_{i j}[m, n]+\boldsymbol{a}_{i j}[m, n] \boldsymbol{v} \boldsymbol{X}_{i j}[m, n] & =\mathbf{0}, \forall i, i \neq j,(15) \\
\sum_{m=1}^{M_{i}} \sum_{n=1}^{N_{i}} \boldsymbol{B}_{i i}[m, n]+\boldsymbol{a}_{i i}[m, n] \boldsymbol{v} \boldsymbol{X}_{i i}[m, n] & =\boldsymbol{I}, \forall i
\end{aligned}
$$




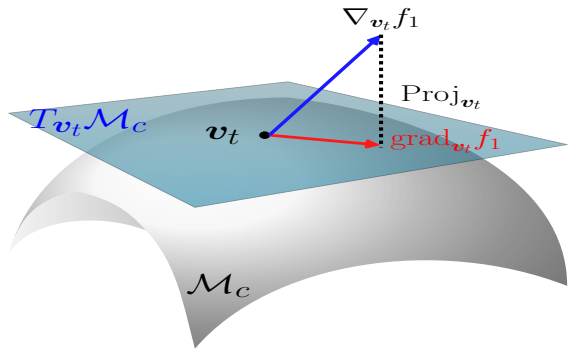

(a) Tangent space and Riemannian gradient

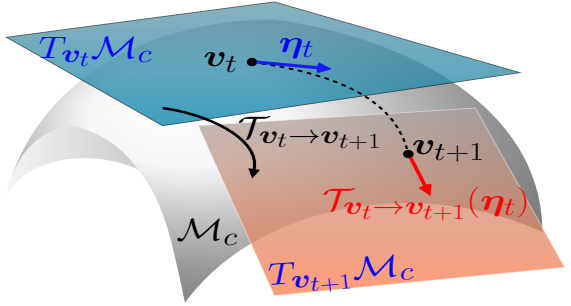

(b) Vector Transport

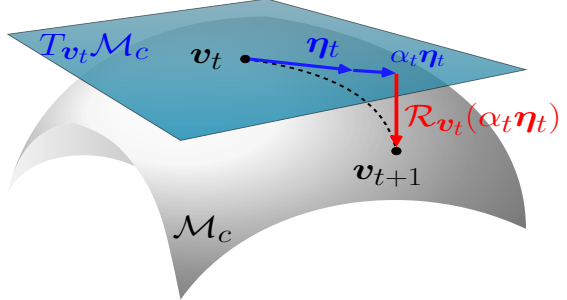

(c) Retraction

Fig. 1. An illustrative example of the key steps in manifold optimization.

By vectorizing (15) and (16), we have

$$
\begin{gathered}
\operatorname{Vec}\left(\sum_{m=1}^{M_{i}} \sum_{n=1}^{N_{j}} \boldsymbol{B}_{i j}[m, n]+\boldsymbol{a}_{i j}[m, n] \boldsymbol{v} \boldsymbol{X}_{i j}[m, n]\right) \\
=\left(\sum_{m=1}^{M_{i}} \sum_{n=1}^{N_{j}}\left(\operatorname{Vec}\left(\boldsymbol{X}_{i j}[m, n]\right) \otimes \boldsymbol{a}_{i j}[m, n]\right)\right) \boldsymbol{v}+ \\
\quad \sum_{m=1}^{M_{i}} \sum_{n=1}^{N_{j}} \operatorname{Vec}\left(\boldsymbol{B}_{i j}[m, n]\right) .
\end{gathered}
$$

Hence, problem (13) can be formulated as follows

$$
\begin{array}{r}
\mathscr{P}_{1}: \underset{\boldsymbol{v}}{\operatorname{minimize}} \\
f_{1}(\boldsymbol{v})=\frac{1}{2}\|\boldsymbol{A} \boldsymbol{v}-\boldsymbol{c}\|_{2}^{2} \\
\text { subject to }\left|v_{l}\right|=1, \forall l=1, \ldots, L,
\end{array}
$$

where

$$
\begin{aligned}
\boldsymbol{A}_{i j} & =\sum_{m=1}^{M_{i}} \sum_{n=1}^{N_{j}}\left(\operatorname{Vec}\left(\boldsymbol{X}_{i j}[m, n]\right) \otimes \boldsymbol{a}_{i j}[m, n]\right) \in \mathbb{C}^{d_{i} d_{j} \times L}, \\
\boldsymbol{A} & =\left[\boldsymbol{A}_{11} ; \boldsymbol{A}_{12} ; \ldots ; \boldsymbol{A}_{i j} ; \ldots ; \boldsymbol{A}_{K K}\right] \in \mathbb{C}^{S \times L}, \\
\boldsymbol{e}_{i j} & =\sum_{m=1}^{M_{i}} \sum_{n=1}^{N_{j}} \operatorname{Vec}\left(\boldsymbol{B}_{i j}[m, n]\right) \in \mathbb{C}^{d_{i} d_{j} \times 1}, \\
\boldsymbol{e} & =\left[\boldsymbol{e}_{11} ; \ldots ; \boldsymbol{e}_{i j} ; \ldots ; \boldsymbol{e}_{K K}\right] \in \mathbb{C}^{S \times 1}, \boldsymbol{c}=\boldsymbol{b}-\boldsymbol{e} \in \mathbb{C}^{S \times 1} .
\end{aligned}
$$

Problem $\mathscr{P}_{1}$ is a non-convex quadratically constrained quadratic programming. The non-convex unit modulus constraints are the main obstacles of solving $\mathscr{P}_{1}$. Note that $\boldsymbol{v}$ lies on the manifold encoded by product of $L$ circles in the complex plane, denoted as $\mathcal{M}_{c}=\left\{\boldsymbol{v} \in \mathbb{C}^{L}:\left|v_{1}\right|=\cdots=\right.$ $\left.\left|v_{L}\right|=1\right\}$ [20]. We aim to develop computationally efficient Riemanian optimization algorithm on the product manifold of $L$ complex circles.

Specifically, the main idea is to generalize a conjugate gradient (CG) method from Euclidean space to manifold space. Similarly, we need to compute search directions in the tangent space and appropriate stepsizes on the manifold. The main steps of the RCG algorithm consists of three steps in each iteration as shown in Fig. 1.

1) Tangent Space and Riemannian Gradient: A tangent space $T_{\boldsymbol{v}_{t}} \mathcal{M}_{c}$ is composed of all the tangent vectors to $\mathcal{M}_{c}$ at any point $\boldsymbol{v}_{t}$ on a manifold. The Riemannian gradient is one tangent vector (direction) with the decrease of the objective function over the manifold space. For the complex circle manifold $\mathcal{M}_{c}$, the tangent space at $\boldsymbol{v}_{t}$ is given by

$$
T_{\boldsymbol{v}_{t}} \mathcal{M}_{c}=\left\{\boldsymbol{z} \in \mathbb{C}^{L}: \Re\left\{\boldsymbol{z} \odot \boldsymbol{v}_{t}^{*}\right\}=\mathbf{0}_{L}\right\} .
$$

As shown in Fig. 1(a), the Riemannian gradient of $f_{1}$ at $\boldsymbol{v}_{t}$, denoted by $\operatorname{grad}_{\boldsymbol{v}_{t}} f_{1}$, can be obtained by orthogonally projecting the Euclidean gradient $\nabla_{\boldsymbol{v}_{t}} f_{1}$ onto the tangent space $T_{\boldsymbol{v}_{t}} \mathcal{M}_{c}$ given by

$$
\operatorname{grad}_{\boldsymbol{v}_{t}} f_{1}=\nabla_{\boldsymbol{v}_{t}} f_{1}-\Re\left\{\nabla_{\boldsymbol{v}_{t}} f_{1} \odot \boldsymbol{v}_{t}^{*}\right\} \circ \boldsymbol{v}_{t},
$$

where the Euclidean gradient of $f_{1}$ at $\boldsymbol{v}_{t}$ is given by

$$
\nabla_{\boldsymbol{v}_{t}} f_{1}=\boldsymbol{A}^{\mathrm{H}}\left(\boldsymbol{A} \boldsymbol{v}_{t}-\boldsymbol{c}\right) .
$$

2) Transport: The search directions $\boldsymbol{\eta}_{t}$ and $\boldsymbol{\eta}_{t+1}$ in manifold optimization generally lie in two different tangent spaces $T_{\boldsymbol{v}_{t}} \mathcal{M}_{c}$ and $T_{\boldsymbol{v}_{t+1}} \mathcal{M}_{c}$, respectively. Therefore, the vector transport $\mathcal{T}_{\boldsymbol{v}_{t} \rightarrow \boldsymbol{v}_{t+1}}\left(\boldsymbol{\eta}_{t}\right)$ for manifold $\mathcal{M}_{c}$ is the map of a tangent vector $\boldsymbol{\eta}_{t}$ from $T_{\boldsymbol{v}_{t}} \mathcal{M}_{c}$ to $T_{\boldsymbol{v}_{t+1}} \mathcal{M}_{c}$ given by, as shown in Fig. 1(b),

$$
\mathcal{T}_{\boldsymbol{v}_{t} \rightarrow \boldsymbol{v}_{t+1}}\left(\boldsymbol{\eta}_{t}\right) \triangleq \boldsymbol{\eta}_{t} \mapsto \boldsymbol{\eta}_{t}-\Re\left\{\boldsymbol{\eta}_{t} \odot \boldsymbol{v}_{t+1}^{*}\right\} \odot \boldsymbol{v}_{t+1} .
$$

Thus, the update rule of the search direction for the RCG is

$$
\boldsymbol{\eta}_{t+1}=-\operatorname{grad}_{\boldsymbol{v}_{t+1}} f_{1}+\beta_{t} \mathcal{T}_{\boldsymbol{v}_{t} \rightarrow \boldsymbol{v}_{t+1}}\left(\boldsymbol{\eta}_{t}\right),
$$

where $\beta_{t}$ is chosen as the Polak-Ribiere parameter [14].

3) Retraction: After determining the search direction $\eta_{t}$ at $\boldsymbol{v}_{t}$ and armijo backtracking line search step size $\alpha_{t}$ that the obtained point $\alpha_{t} \boldsymbol{\eta}_{t}$ does not lie in $\mathcal{M}_{c}$, we need to map it from the tangent space $T_{\boldsymbol{v}_{t}} \mathcal{M}_{c}$ to the manifold $\mathcal{M}_{c}$ by using retraction operator given by, as shown in Fig. 1(c),

$$
\boldsymbol{v}_{t+1}=\mathcal{R}_{\boldsymbol{v}_{t}}\left(\alpha_{t} \boldsymbol{\eta}_{t}\right) \triangleq \alpha_{t} \boldsymbol{\eta}_{t} \mapsto\left(\alpha_{t} \boldsymbol{\eta}_{t}\right) \odot \frac{1}{\left(\alpha_{t} \boldsymbol{\eta}_{t}\right)} .
$$

Now, the key steps used in each iteration of the manifold optimization have been introduced. The resulting algorithm for solving $\mathscr{P}_{1}$ is summarized in Algorithm 2.

\section{Optimizing Block $\boldsymbol{X}$}

For a given phase-shifter matrix $\Theta$, the concatenated channel response $\tilde{\boldsymbol{H}}_{i j}=\boldsymbol{H}_{i j}+\boldsymbol{R}_{i} \boldsymbol{\Theta} \boldsymbol{T}_{j}$ is fixed, and hence problem (13) can be simplified as

$$
\begin{aligned}
& \underset{\boldsymbol{X}}{\operatorname{minimize}} f_{2}(\boldsymbol{X})=\frac{1}{2}\left\|\mathcal{A}_{2}(\boldsymbol{X})-\boldsymbol{b}\right\|_{2}^{2} \\
& \text { subject to } \operatorname{rank}(\boldsymbol{X})=r,
\end{aligned}
$$

where $\mathcal{A}_{2}$ is a linear operator: $\mathbb{C}^{M \times N} \mapsto \mathbb{C}^{S}$ as a function of $\left\{\tilde{\boldsymbol{H}}_{i j}\right\}$. This is a classical low-rank matrix completion (LRMC) problem, which has been studied extensively in the literature using convex and non-convex approaches. The authors in [18] proposed a Riemannian optimization 
algorithm on manifold with better performance and higher computational efficiency compared with that of in Euclidean space for topological cooperation, where the problem (25) is reformulated into the following problem

$$
\mathscr{P}_{2}: \underset{\boldsymbol{Y} \in \mathbb{C}^{N \times r}}{\operatorname{minimize}} f_{2}(\boldsymbol{Y})=\frac{1}{2}\left\|\mathcal{B}_{2}\left(\boldsymbol{Y} \boldsymbol{Y}^{\mathrm{H}}\right)-\boldsymbol{b}\right\|_{2}^{2} \text {. }
$$

where $\boldsymbol{X}=\boldsymbol{L} \boldsymbol{R}^{\mathrm{H}}, \boldsymbol{Y}=[\boldsymbol{L} ; \boldsymbol{R}] \in \mathbb{C}^{(M+N) \times r}$ and $\mathcal{B}_{2}\left(\boldsymbol{Y} \boldsymbol{Y}^{\mathrm{H}}\right)=\mathcal{A}_{2}\left(\boldsymbol{L} \boldsymbol{R}^{\mathrm{H}}\right)$. This is a Riemannian optimization problem with a smooth objective function over the complex non-compact Stiefel manifold $\mathcal{M}_{r}=\left\{\boldsymbol{Y} \in \mathbb{C}^{(M+N) \times r}\right.$ : $\operatorname{rank}(\boldsymbol{Y})=r\}$, i.e., the set of all $N \times r$ full column rank matrices in complex field. Similar to Algorithm 2, the details of the RCG algorithm for the Problem $\mathscr{P}_{2}$ are shown in [18]. We use the manifold optimization toolbox Manopt [20] to implement the proposed RCG algorithms.

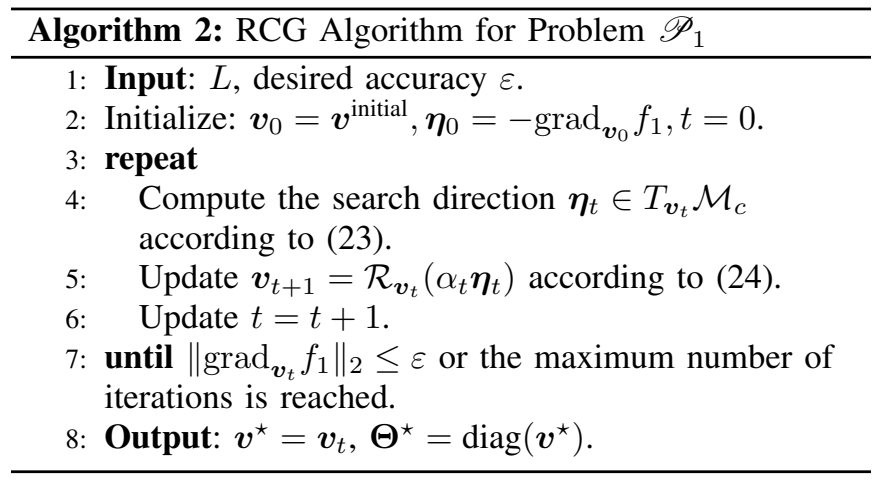

\section{NUMERICAL RESULtS}

In this section, we present the numerical results of the proposed block-structured Riemannian pursuit method in RIS-assisted MIMO D2D networks. We assume that RIS is placed at $(25,20)$ meters. The transmitters and receivers are randomly distributed in the region of $(0,20) \times(0,20)$ meters and $(30,50) \times(0,20)$ meters, respectively, the path loss model is $L(d)=T_{0}(d)^{-\alpha}$, where $T_{0}=-30 \mathrm{~dB}$ is the path loss at reference distance, $d$ is the link distance, and $\alpha$ is the path loss exponent. The path loss exponents for the useruser link, the transmitter-RIS link, and the RIS-receiver link are set to $2.8,2$, and 2 , respectively [8]. We denote $d_{\mathrm{RT}}^{i j}$, and $d_{\mathrm{IR}}^{i}, d_{\mathrm{IT}}^{j}$ as the distances between the $i$-th receiver and the $j$-th transmitter, between the $i$-th receiver and the RIS, and between the $j$-th transmitter and the RIS, respectively. All channels are assumed to suffer from Rician fading [8]. Hence, the corresponding channel coefficients are

$$
\boldsymbol{H}_{i j}=\sqrt{L\left(d_{\mathrm{RT}}^{i j}\right)}\left(\sqrt{\frac{\beta_{R T}}{1+\beta_{R T}}} \boldsymbol{H}_{i j}^{\mathrm{LOS}}+\sqrt{\frac{1}{1+\beta_{R T}}} \boldsymbol{H}_{i j}^{\mathrm{NLOS}}\right),
$$

where $\beta_{R T}$ is the Rician factor, and $\boldsymbol{H}_{i j}^{\mathrm{LOS}}$ and $\boldsymbol{H}_{i j}^{\mathrm{NLOS}}$ denote the deterministic LoS and Rayleigh fading components, respectively. The Rician factors of the RIS-receiver link and the transmitter-RIS link are denoted by $\beta_{I R}$ and $\beta_{I T}$, respectively. All transmitters and receivers are equipped with $M_{1}$ and $N_{1}$ antennas, respectively. We set the number of data streams as $d_{i}=N_{1}, \forall i \in \mathcal{K}$. We set $\sigma^{2}=-120$ $\mathrm{dB}, \epsilon=10^{-4}, \varepsilon=10^{-10}, T=30, K=10$, and $\beta_{I R}=\beta_{I T}=\beta_{R T}=10$.

For performance comparison, we consider the following schemes in simulations: 1) Block-structured RCG: Joint transceiver and phase-shift matrices design by RCG proposed in Section III. 2) Block-structured GD: Joint transceiver and phase-shift matrices by gradient descent algorithm (GD) given in [18]. 3) Benchmark scheme with random phase shifts: Randomly choose the phase shifts. 4) Benchmark scheme without RIS.

Fig. 2(a) plots the achievable DoF versus the number of receive antennas $M_{1}$ when $N_{1}=2$ and $L=150$. First, we observe that deploying more antennas at the receiver gives rise to the achievable DoF for all algorithms, due to the higher diversity and power gains. Furthermore, joint transceiver and phase-shift matrices significantly outperforms random phase shifts schemes. It demonstrates the necessity of optimizing the phase-shifter matrix at the RIS, which enhance the conditionedness of the composite channel matrices, thereby improving the feasibility of interference alignment conditions. In addition, due to the superiority of the proposed RCG algorithms, the block-structured RCG algorithm can achieve higher DoFs than the block-structured GD algorithm.

In Fig. 2(b), we investigate the achievable sum rate performance over different SNR values when $N_{1}=2, M_{1}=6$, and $L=150$. We observe that the sum rate can also be increased by jointly designing transceiver and phase-shift matrices, due to the DoF gain. In addition, we observe that the blockstructured RCG algorithm outperforms the block-structured GD algorithm in terms of the achievable sum rate.

In Fig. 2(c), we plot the achievable DoFs versus the Rician factor of the transmitter-receiver channels $\beta_{R T}$ when $N_{1}=M_{1}=4$, and $L=50$ by using proposed algorithms shown in Section III. One can observe that when $\beta_{R T}$ increases, the achievable DoFs of the schemes without RIS fall sharply. This is because for schemes without RIS, a higher Rician factor of $\boldsymbol{H}_{i j}$ results in rank-deficient MIMO channels, which leads to spatial multiplexing gain decrease. However, we observe that deploying an RIS make the achievable DoFs decrease slowly as $\beta_{R T}$ increase. This result is due to the fact that the reflected path by deploying the RIS makes angular separation at the receiver and thus provides DoF gain. Furthermore, the achievable DoFs can be further enhanced to jointly design transceiver and phase-shift matrices by using the block-structured RCG algorithm even when the number of passive reflecting elements is small. The implication of this result is that it is favorable to optimize the phase shifters to compensate the direct-link rank-deficient MIMO channels, so as to serve more multiple pairs that require sufficient DoFs compared to conventional D2D networks.

Fig. 2(d) illustrates the effect of the number of passive reflecting elements at the RIS (i.e., $L$ ) on the sum rate when $N_{1}=M_{1}=4$ and $\mathrm{SNR}=130 \mathrm{~dB}$. The achievable sum rate increases as the value of $L$ increases by jointly designing the transceiver matrix and phase-shift matrix. This result suggests 


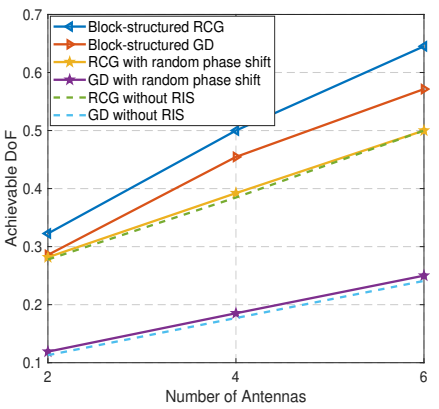

(a) Achievable DoF vs. The number of receive antennas $M_{1}$.

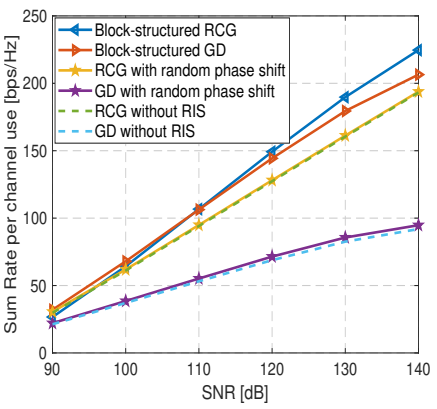

(b) Achievable
the transmitter

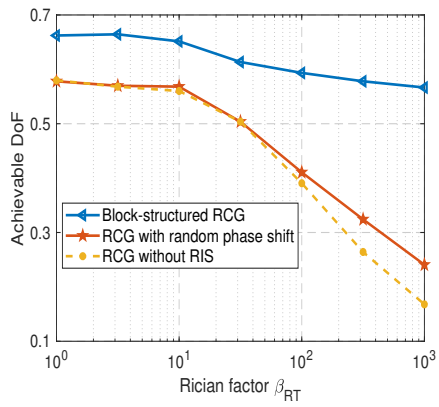

(c) Achic
$\beta_{R T}$

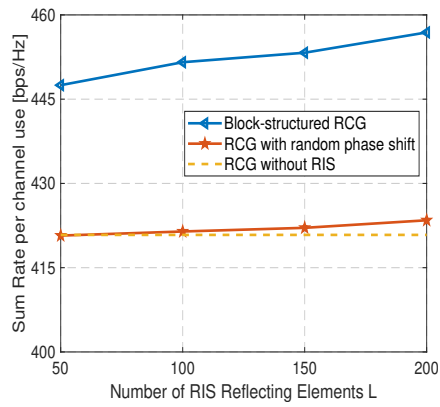

(d) Achievable sum rate vs. The number of RIS elements $L$.

Fig. 2. Performance comparisons between the proposed method and state-of-the-art methods under different network settings.

the sum rate should be further improved by appropriately setting the number of RIS elements while decreasing the transmitted power.

\section{CONCLUSION}

In this paper, we considered an RIS-assisted MIMO D2D networks, where the RIS assists the interference alignment to alleviate the co-channel interference among multiple transceiver pairs. We exploited an RIS yielding compensation for direct-link rank-deficient channels to improve the feasibility of interference alignment conditions, thereby increasing the achievable DoFs. Specifically, we presented a rank minimization to maximize DoFs by designing the phase-shift matrix at RIS, transceiver matrix while satisfying interference alignment conditions. To achieve the algorithmic advantages and admirable performance, a block-structured Riemannian pursuit algorithm was developed. This is achieved by performing a sequence of non-convex least square problems with rank increase, followed by unified RCG algorithms to alternately design one block of the fixed-rank transceiver matrix and the unit modulus constrained phase shifters. Simulation results showed the effectiveness of deploying an RIS and the superiority of the proposed block-structured Riemannian pursuit in terms of the achievable DoFs and sum rate.

\section{REFERENCES}

[1] A. Asadi, Q. Wang, and V. Mancuso, "A survey on device-to-device communication in cellular networks," IEEE Commun. Surveys Tuts., vol. 16, no. 4, pp. 1801-1819, 4th quart. 2014.

[2] K. Shen, W. Yu, L. Zhao, and D. P. Palomar, "Optimization of MIMO device-to-device networks via matrix fractional programming: A minorizationmaximization approach," IEEE/ACM Trans. Netw., vol. 27, no. 5, pp. 2164-2177, Oct. 2019.

[3] G. Sridharan and W. Yu, "Linear beamformer design for interference alignment via rank minimization," IEEE Trans. Signal Process., vol. 63, no. 22, pp. 5910-5923, Jul. 2015.

[4] S. R. Krishnamurthy, A. Ramakrishnan, and S. A. Jafar, "Degrees of freedom of rank-deficient MIMO interference channels," IEEE Trans. Inf. Theory, vol. 61, no. 1, pp. 341-365, Jan. 2015.

[5] X. Yuan, Y.-J. Zhang, Y. Shi, W. Yan, and H. Liu, "Reconfigurableintelligent-surface empowered $6 \mathrm{G}$ wireless communications: Challenges and opportunities," arXiv preprint arXiv:2001.00364, 2020.

[6] Q. Wu and R. Zhang, "Towards smart and reconfigurable environment: Intelligent reflecting surface aided wireless network," IEEE Commun. Mag., vol. 58, no. 1, pp. 106-112, Jan. 2020.

[7] K. Yang, Y. Shi, Y. Zhou, Z. Yang, L. Fu, and W. Chen, "Federated machine learning for intelligent IoT via reconfigurable intelligent surface," arXiv preprint arXiv:2004.05843, 2020.
[8] Q. Wu and R. Zhang, "Intelligent reflecting surface enhanced wireless network via joint active and passive beamforming," IEEE Trans. Wireless Commun., vol. 18, no. 11, p. 53945409, Aug. 2019.

[9] M. Fu, Y. Zhou, and Y. Shi, "Intelligent reflecting surface for downlink non-orthogonal multiple access networks," in Proc. IEEE Global Commun. Conf. (Globecom) Workshops, Waikoloa, HI, Dec. 2019.

[10] C. Huang, A. Zappone, G. C. Alexandropoulos, M. Debbah, and C. Yuen, "Reconfigurable intelligent surfaces for energy efficiency in wireless communication," IEEE Trans. Wireless Commun., vol. 18, no. 8, pp. 4157-4170, Aug. 2019.

[11] H. Guo, Y. Liang, J. Chen, and E. G. Larsson, "Weighted sumrate maximization for reconfigurable intelligent surface aided wireless networks," IEEE Trans. Wireless Commun., vol. 19, no. 5, pp. 30643076, May 2020.

[12] X. Yu, D. Xu, and R. Schober, "MISO wireless communication systems via intelligent reflecting surfaces," in in Proc. IEEE/CIC Int. Conf. Commun. China (ICCC). IEEE, Changchun, China, Aug. 2019.

[13] S. Xia and Y. Shi, "Intelligent reflecting surface for massive device connectivity: Joint activity detection and channel estimation," in Proc. IEEE Int. Conf. Acoust. Speech Signal Process. (ICASSP), Barcelona, Spain, May 2020.

[14] P. A. Absil, R. Mahony, and R. Sepulchre, Optimization Algorithms on Matrix Manifolds. Princeton, NJ, USA: Princeton Univ. Press., 2009.

[15] J. Hu, X. Liu, Z. Wen, and Y. Yuan, A Brief Introduction to Manifold Optimization. China, J. Oper. Res. Soc., 2020, doi 10.1007/s40305020-00295-9.

[16] Y. Shi, J. Zhang, and K. B. Letaief, "Low-rank matrix completion for topological interference management by riemannian pursuit," IEEE Trans. Wireless Commun., vol. 15, no. 7, pp. 4703-4717, Jul. 2016.

[17] Y. Shi, B. Mishra, and W. Chen, "Topological interference management with user admission control via riemannian optimization," IEEE Trans. Wireless Commun., vol. 16, no. 11, pp. 7362-7375, Nov. 2017.

[18] K. Yang, Y. Shi, and Z. Ding, "Generalized low-rank optimization for topological cooperation in ultra-dense networks," IEEE Trans. Wireless Commun., vol. 18, no. 5, pp. 2539-2552, May 2019.

[19] J. Dong, Y. Kai, and Y. Shi, "Blind demixing for low-latency communication," IEEE Trans. Wireless Commun., vol. 18, no. 2, pp. 897-911, Feb. 2019.

[20] N. Boumal, B. Mishra, P. A. Absil, and R. Sepulchre, "Manopt, a MATLAB toolbox for optimization on manifolds," J. Mach. Learn. Res., vol. 15, p. 14551459, Jan. 2014. 